\title{
Carbon nanotube electrodes for effective interfacing with retinal tissue
}

\author{
Asaf Shoval' ${ }^{1}$ Christopher Adams ${ }^{2}$, Moshe David-Pur ${ }^{1}$, Mark Shein ${ }^{1}$, Yael Hanein ${ }^{1 *}$ and Evelyne Sernagor ${ }^{2}$ \\ School of Electrical Engineering, Tel-Aviv University, Tel-Aviv, Israel \\ 2 Institute of Neuroscience, Medical School, Newcastle University, Newcastle, UK
}

Edited by:

Fabrizio Gelain, University of Milano-

Bicocca, Italy

A.O. Niguarda Ca'Grande, Italy

\section{Reviewed by:}

Claudio Punzo, Harvard Medical School, Harvard University, USA

S. Krishnakumar, Vision Research

Foundation Sankara Nethralaya, India

Elisha Moses, Weizmann Institute,

Israel

\section{*Correspondence:}

Yael Hanein, School of Electrical

Engineering, Tel-Aviv University, Tel-Aviv

69978, Israel.

e-mail: hanein@eng.tau.ac.il
We have investigated the use of carbon nanotube coated microelectrodes as an interface material for retinal recording and stimulation applications. Test devices were micro-fabricated and consisted of 60, $30 \mu \mathrm{m}$ diameter electrodes at spacing of $200 \mu \mathrm{m}$. These electrodes were coated via chemical vapor deposition of carbon nanotubes, resulting in conducting, three dimensional surfaces with a high interfacial area. These attributes are important both for the quality of the cell-surface coupling as well as for electro-chemical interfacing efficiency. The entire chip was packaged to fit a commercial multielectrode recording and stimulation system. Electrical recordings of spontaneous spikes from whole-mount neonatal mouse retinas were consistently obtained minutes after retinas were placed over the electrodes, exhibiting typical bursting and propagating waves. Most importantly, the signals obtained with carbon nanotube electrodes have exceptionally high signal to noise ratio, reaching values as high as 75. Moreover, spikes are marked by a conspicuous gradual increase in amplitude recorded over a period of minutes to hours, suggesting improvement in cell-electrode coupling. This phenomenon is not observed in conventional commercial electrodes. Electrical stimulation using carbon nanotube electrodes was also achieved. We attribute the superior performances of the carbon nanotube electrodes to their three dimensional nature and the strong neuro-carbon nanotube affinity. The results presented here show the great potential of carbon nanotube electrodes for retinal interfacing applications. Specifically, our results demonstrate a route to achieve a reduction of the electrode down to few micrometers in order to achieve high efficacy local stimulation needed in retinal prosthetic devices.

Keywords: carbon nanotube, retina, neural implant, biocompatibility, multielectrode array

\section{INTRODUCTION}

In recent years, multielectrode arrays (MEAs) have become a widespread tool for interfacing with neural assemblies, both for stimulation and recording purposes. Moreover, they are now recognized as one of the main approaches for the design of neural prosthetic devices to artificially restore impaired neural function (e.g. for vision, hearing and limb movement). With the exception of cochlear implants, this emerging technology does, however, still face tremendous challenges before these interfaces will become realistically useful as neural implants. One key issue is the biocompatibility of the electrode-tissue interface which must not only allow good electrical recording and stimulation performance, but also encourage strong cell adhesion and proliferation so that the electrodes remain effectively long-term coupled with the neurons. For optimal electrical coupling, the electrode surface must be as rough as possible so that it effectively enlarges the surface area. Independently, to achieve good biological coupling, the surface must be cell-adhesive. Interestingly, these two requirements can be attained concurrently if extremely rough and conducting surfaces are used (Gabay et al., 2007; Sorkin et al., 2006). Such surfaces can function both as excellent electro-chemical electrodes and substrates for neuronal growth. Using this novel approach it is possible to directly interface with neurons in an unmediated fashion (Shein et al., 2009).
Carbon nanotubes (CNTs) appear to be a most suitable interface material for these applications because they are chemically inert, robust against mechanical damage and easy to produce. In addition, high density CNT-coated surfaces are characterized by having an extremely high surface area. Most interestingly, CNTs appear to encourage neuronal growth. Finally, since CNTs are good electrical conductors, they may also function as very effective electrochemical electrodes. Resulting from this unique combination of properties, CNT electrodes offer exciting new opportunities as an interface material for neuronal applications. This has now been validated by several recent studies (Gabay et al., 2007; Hu et al., 2004, 2005; Malarkey and Parpura, 2007; Mattson et al., 2000; Sorkin et al., 2006).

One medical application that may particularly benefit from this technology is retinal implants. Vision loss due to retinal degeneration (e.g. Leber Congenital Amaurosis; den Hollander et al., 2008 or age-related macular degeneration; Ehrlich et al., 2008) is a worldwide impairment in ageing populations, often leading to complete blindness. The common phenotype of these retinal dystrophies is photoreceptor degeneration, whilst retinal ganglion cells (RGCs), the output cells of the retina, remain intact and can still transmit information to the visual centers of the brain. Consequently, a plethora of projects aiming at developing retinal implantable prosthetic devices to stimulate RGCs directly, circumventing the 
degenerated photoreceptor pathway, have emerged in recent years (Rizzo et al., 2007). The results are indicative of the viability of MEA stimulation, but this technology is still very much in its infancy (Dowling, 2008; Mahadevappa et al., 2005; Rizzo et al., 2003). The present limited success arises from many technical challenges, ranging from biocompatibility and intraocular implant stability, to problems of stimulation efficacy and restriction of current spread across the retina following focal stimulation. Whilst an optimal device would have a 1:1 ratio of electrode to RGCs, most of the devices currently under development comprise arrays of as few as 12-16 electrodes, each with diameters of several hundred micrometers, thereby covering large retinal areas. Recent studies indicate significant improvement in spatial resolution (MEAs comprising 61 ITO electrodes; spacing $60 \mu \mathrm{m}$, diameters 6-25 $\mu \mathrm{m}$ ) (Gunning et al., 2005; Sekirnjak et al., 2006). It is estimated that an array of 625 electrodes with center-center spacing of $20 \mu \mathrm{m}$ would restore enough visual acuity to enable the patient to navigate their surroundings without additional external aids.

It is evident from many recent studies in the field that a key challenge in developing better MEAs for retinal implant devices is to achieve better coupling between the electrodes and the tissue. This, in turn, will allow the reduction of the electrode size and increase the electrode density on the device.

In this paper, we examined the suitability and the possible advantages of CNT MEAs for retinal implant applications. Using electrophysiological recordings from retinal whole mounts, we bring evidence, for the first time, that CNT electrodes lead to better electrode-tissue coupling, resulting in remarkable signal-to-noise ratio.

\section{MATERIALS AND METHODS CNT MEA}

CNT MEAs were produced and packaged as reported previously (Gabay et al., 2007). Briefly, TiN electrodes were micro-fabricated on oxidized silicon or quartz substrates and were then passivated with $\mathrm{SiN}$ and coated with CNTs using chemical vapour deposition. CNTs grown are multi wall carbon nanotubes (MWCNTs). CNT diameters, estimated from scanning electron microscope imaging (Sorkin et al., 2009), average around $82 \mathrm{~nm}$ with very narrow distribution. CNT length is several microns. Moreover, energy-dispersive $\mathrm{X}$-ray (EDX) analysis was carried out and reported in Gabay et al. (2007) and revealed no measurable quantities of the catalyst. Amorphous carbon may be present in our electrodes, and can be reduced, for long term, applications by varying growth parameters and post-growth thermal treatment (Wang et al., 2006). Samples were diced prior to growth to fit into a custom designed circuit board (PCB). After CNT growth, samples were mounted and glued to the PCB. A shallow quartz ring was glued to the PCB-silicon assembly to form a well to accommodate the artificial cerebrospinal fluid. The quartz ring was glued using a thin disk of polydimethylsiloxane (PDMS), which was shaped to accommodate the siliconPCB and to provide leak-tight seal against liquid.

\section{TISSUE PREPARATION AND RECORDINGS}

This study was done using C57bl/6 neonatal mice. All animal procedures were conducted under the UK Home Office, Animals (Scientific procedures) Act 1986. As described elsewhere (Hennig et al., 2009), mouse pups were killed by cervical dislocation and enucleated prior to retinal isolation. The isolated retina was then transferred to the experimental chamber and placed, RGC layer facing down, onto MEAs consisting either of 60 titanium nitride (TiN) electrodes (30 $\mu \mathrm{m}$ diameter, $200 \mu \mathrm{m}$ spacing) arranged in an $8 \times 8$ grid on indium tin oxide substrate (MultiChannel Systems, Reutlingen, Germany) or of 60 CNT electrodes (30 $\mu$ m diameter, $200 \mu \mathrm{m}$ spacing) in an $8 \times 8$ grid on silicon substrate. Better coupling between the tissue and the electrodes was achieved by placing a small piece of polyester membrane filter ( $5 \mu \mathrm{m}$ pores) (Sterlitech, Kent, WA, USA) on the retina followed by a slice anchor holder. Retinas were kept at $32^{\circ} \mathrm{C}$ and continuously perfused $(2-5 \mathrm{ml} / \mathrm{min}$ ) with artificial cerebrospinal fluid containing the following (in $\mathrm{mM}$ ): $118 \mathrm{NaCl}, 25 \mathrm{NaHCO}_{3}, 1 \mathrm{NaH}_{2} \mathrm{PO}_{4}, 3 \mathrm{KCl}, 1 \mathrm{MgCl}_{2}, 2 \mathrm{CaCl}_{2}$, and 10 glucose, equilibrated with $95 \% \mathrm{O}_{2}$ and $5 \% \mathrm{CO}_{2}$. It is standard in vitro procedure to keep brain slices or isolated retinas around $32^{\circ} \mathrm{C}$ rather than $37^{\circ} \mathrm{C}$. Keeping them at higher temperature shortens the time recordings can be performed.

\section{DATA ACQUISITION}

Signals were amplified (gain $\times 1,200$ ) and acquired using a 128-channel analogue to digital converter (MultiChannel Systems MC_Card, Reutlingen, Germany). Signals were digitized at $25 \mathrm{kHz}$ and acquired with $80 \mathrm{~Hz}$ high pass filtering using the software MC_Rack (MultiChannel Systems, Reutlingen, Germany). Once the retina was laid on the MEA, neural activity was sampled during 2-5 min at intervals ranging between 2-20 min. Typically the intervals were shorter at the beginning of the recording session, when changes in coupling occur fast, reflected by an increase in signal amplitude.

\section{DATA ANALYSIS}

Spikes were threshold-detected with MC_Rack (the typical threshold was at signal amplitude that is three times above the RMS baseline noise) so that the firing on each electrode reflects the overall activity level generated by all RGCs on that same electrode (typically up to five cells). Once threshold-detected, spikes were additionally screened according to the method described in Hulata et al. (2002). This method uses wavelet based spike detection and is used to remove non-biological spike-like data. Only electrodes yielding spontaneous activity equal or above $0.2 \mathrm{~Hz}$ were taken into consideration for further analysis.

\section{RESULTS}

Figure 1A shows a CNT MEA on silicon substrate. Note that there are more CNT islands than electrodes. These extra CNT islands are used to assist in stabilizing the dissociated neuronal culture (Shein et al., 2009) or in this case, the whole tissue. Figure 1B shows a high magnification $(\times 9,500)$ micrograph of one CNT island, revealing its highly porous nature. Retinas were mounted on MEAs immediately after isolation. Figure 1C illustrates a retina flattened on a CNT (30 $\mu \mathrm{m}$ CNT islands on $50 \mu \mathrm{m}$ TiN support) array on quartz. The picture was taken from underneath the array using an inverted microscope (20× magnification). The cellular layer in focus with the CNT islands is the RGC layer and the arrow shows the edge of the retina. Figure 1D was taken at higher magnification $(32 \times)$ and clearly reveals well delineated RGC bodies (the arrow points 

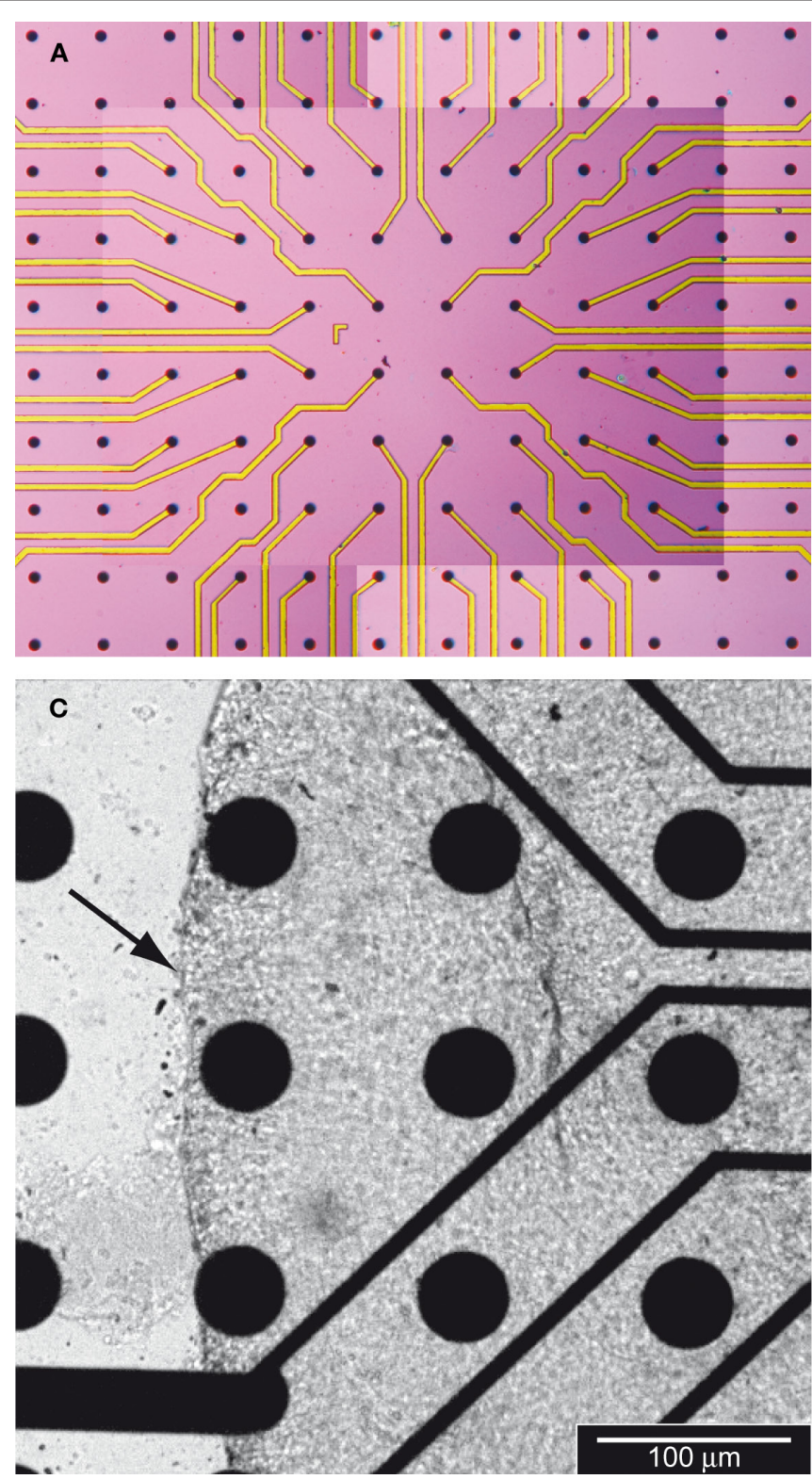

FIGURE 1 | Carbon nanotube MEAs for retinal interfacing and recording. (A) General view of a CNT MEA. Each black dot is a CNT island. All 60 islands in the center of the field are electrodes whilst those at the periphery are not. (B) Micrograph of one CNT island (9,500x magnification) revealing the porous nature of the CNTs. Note that the island in Figure $1 \mathrm{~B}$ is a smaller island than

to one RGC) as well as retinal blood vessels (asterisks). Previous studies on dissociated neurons have demonstrated high cell affinity to these porous CNT coatings (Gabay et al., 2007; Hu et al., 2004, 2005; Malarkey and Parpura, 2007; Mattson et al., 2000; Sorkin et al., 2006), suggesting that CNTs have the potential for high biocompatibility for intact neural tissue such as the retina.

To compare how the retina interacts with CNT electrodes versus commercially available electrodes made of more conventional material, we have recorded RGC signals both from TiN-electrodes MEAs and from CNT-electrodes MEAs. Commercial TiN electrodes are porous by design to achieve high surface area and low impedance
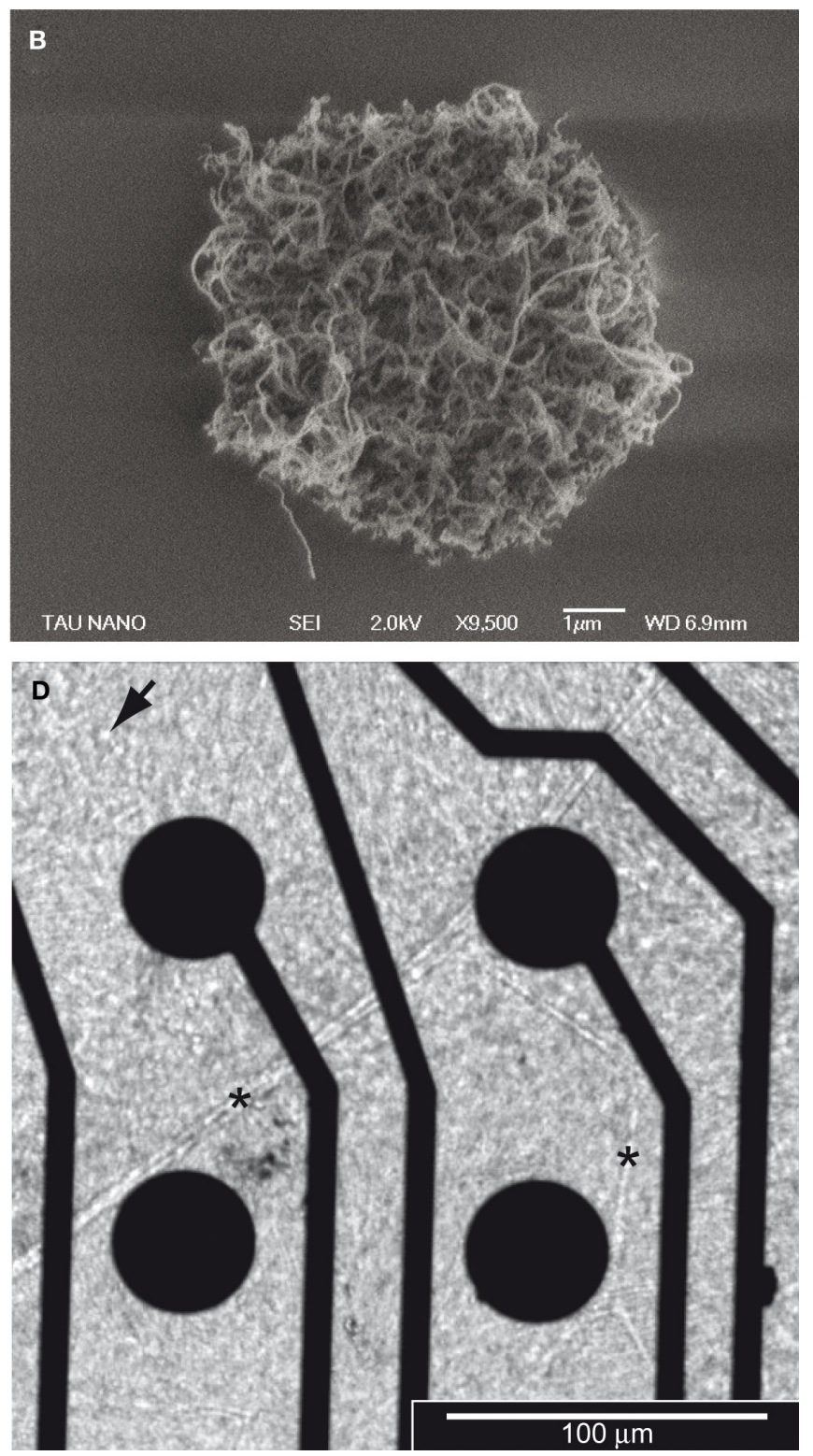

those used in the CNT MEA configuration. (C) Micrograph (20x magnification) of a retina laid on a CNT MEA. The retina is flattened on the CNTs, with the RGC layer facing down, hence in contact with the CNTs. The arrow indicates the edge of the tissue. (D) Micrograph (32× magnification) of the same retina as in (C). The arrow points to a RGC body and the asterisks to blood vessels.

(Janders et al., 1996) with similar electrochemical properties to our CNT MEAs (Gabay et al., 2007) and they can therefore serve as a good platform for comparison. A systematic comparison between the electrochemical properties of CNT and commercial TiN electrodes is presented and discussed in Gabay et al. (2007). Activity was recorded from retinas isolated from 2- to 3-week-old pups, when the retina has high levels of spontaneous activity, with RGCs firing in bursts of action potentials (Hennig et al., 2009; Sernagor et al., 2001). Figure 2 illustrates RGC spontaneous activity recorded from a TiN electrode (Figure 2A) and from a CNT electrode (Figure 2B). Both types of electrodes reveal action potential waveforms. Both 


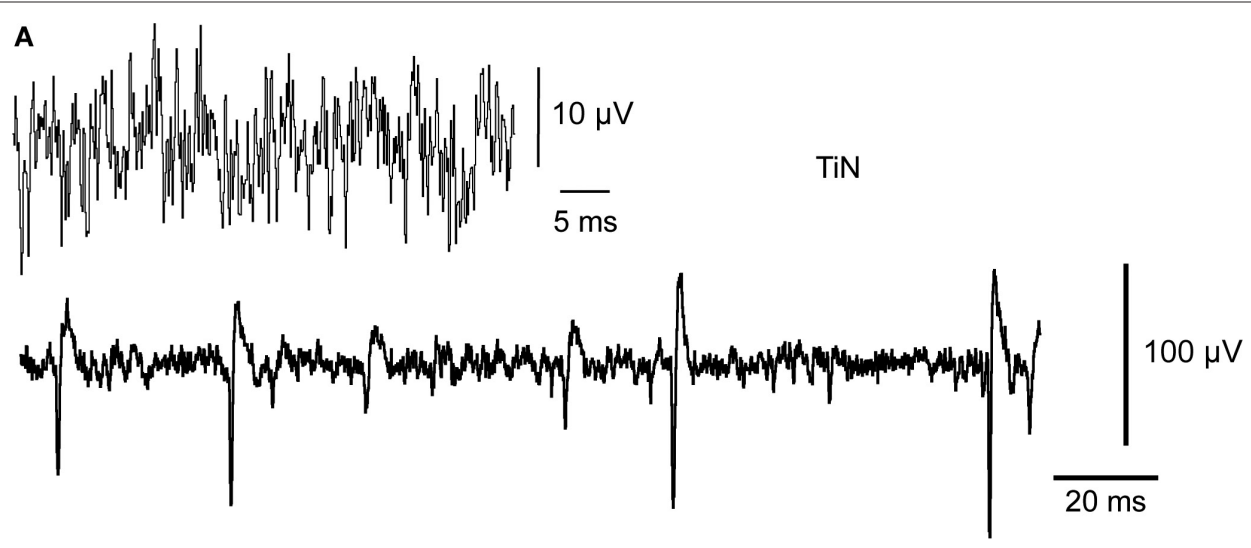

B

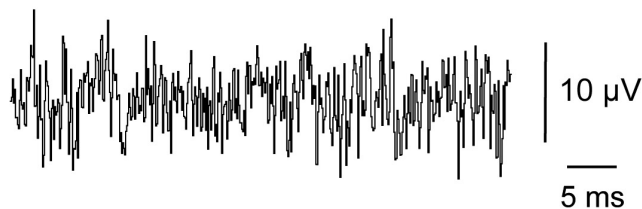

CNT

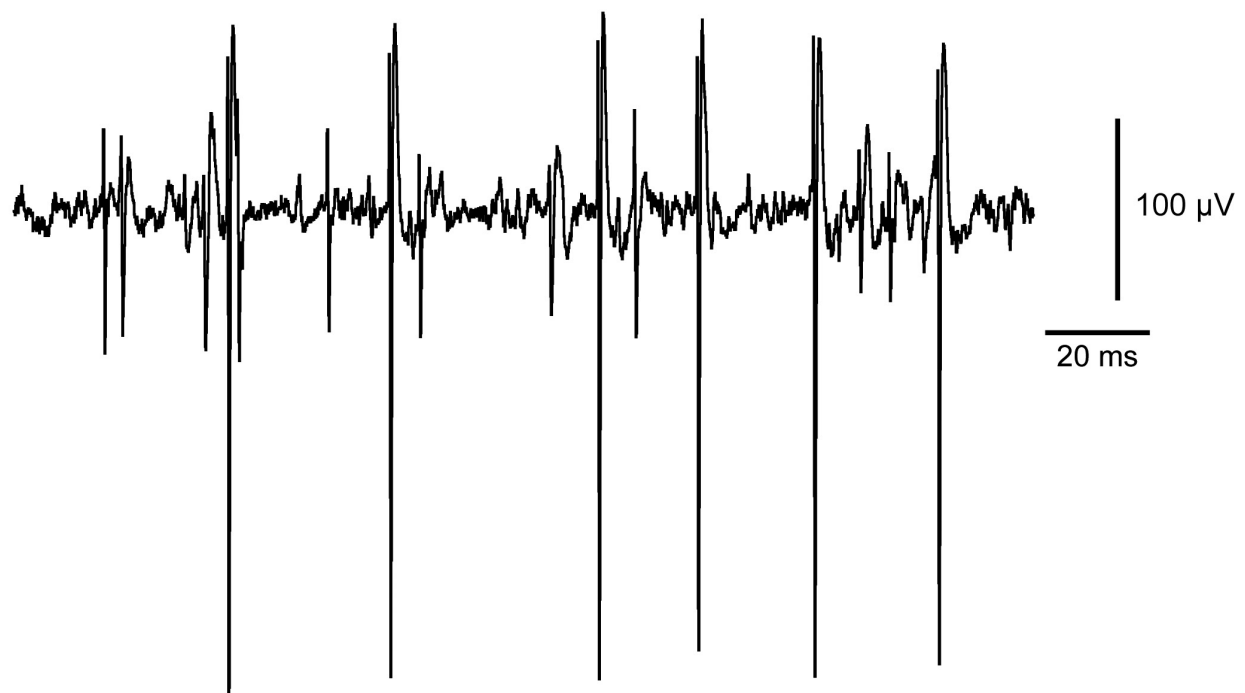

FIGURE 2 | Recordings of neural activity with CNT electrodes yield higher signal-to-noise ratio and amplitudes than commercial electrodes do. (A) Recordings obtained with a TiN electrode. The upper trace shows the typical unfiltered baseline noise level. The lower trace illustrates spontaneous spiking activity. The baseline noise is about $\pm 10 \mu \mathrm{V}$. The largest spikes are about $100 \mu \mathrm{V}$ in amplitude. (B) Recordings obtained with a CNT electrode. The larger spikes (lower trace) have amplitudes reaching up to nearly $300 \mu \mathrm{V}$.

(Figure 3A) and two CNT electrodes (Figure 3B) recorded about $1 \mathrm{~h}$ after mounting the tissue on the MEAs (both electrodes in each case are from the same MEA, hence recording from the same retina). The overall activity shown in each panel of Figure 3 is similar, with a total of 500-700 spikes. Indeed there is no conspicuous difference between the activity rates of retinas on TiN and $\mathrm{CNT}$ electrodes. However, there is variability in the activity rate between different recording sites and between retinas (data not shown). Indeed, spontaneous activity patterns change dramatically over very short periods of time in the developing retina, switching from propagating waves (present from postnatal day 0-12) to scattered bursting activity that can occasionally be extremely strong 

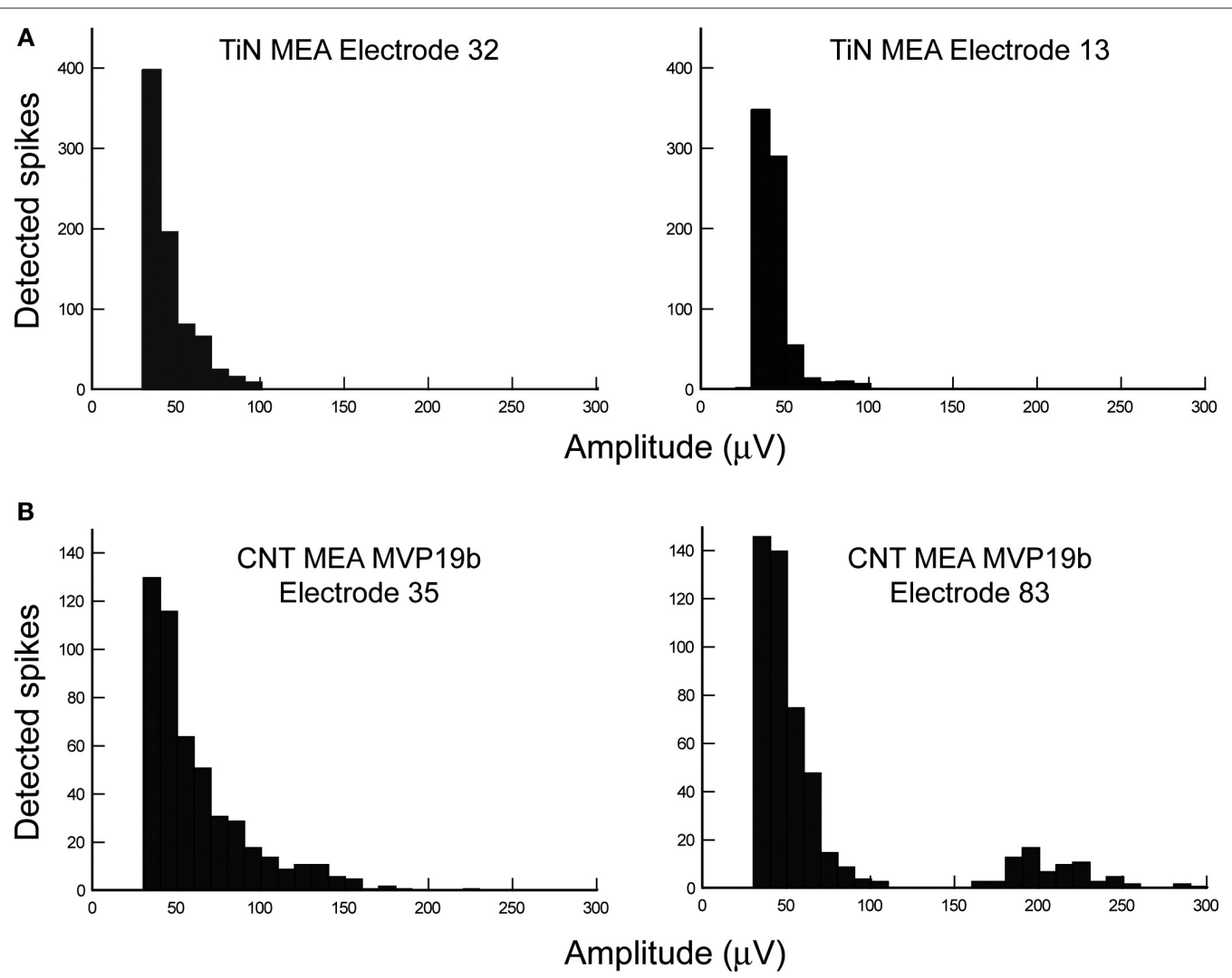

FIGURE 3 | Comparison of spike amplitude distributions recorded with commercial versus CNT electrodes. (A) Histograms of spike amplitudes recorded from two TiN electrodes on the same MEA. Both distributions look relatively similar, with maximal amplitudes of about $100 \mu \mathrm{V}$. (B) Histograms of spike amplitudes recorded from two CNT electrodes on the same MEA. The amplitude distributions are broader than with TiN electrodes, starting from similar values $(25-35 \mu \mathrm{V})$, but reaching much higher values $(175 \mu \mathrm{V}$ for electrode 35 and $290 \mu \mathrm{V}$ for Electrode 83). (postnatal day 12-30). Moreover, there is also variability in activity with retinal eccentricity. Signals recorded from CNT electrodes can reach amplitude values that are more than twice as high as those recorded with $\mathrm{TiN}$ electrodes. It is interesting to notice that the higher values are not related to the total number of recorded spikes. Indeed, there was more neural activity in the retina used with both TiN electrodes than in the retina used with the CNT electrodes. Nevertheless, the TiN electrodes did not record particularly large signals. It is also important to note that the high amplitude signals are single unit recordings (as can be clearly seen in Figure 2B) so, for the most part, the amplitude increase is not due to superposition of simultaneous recordings from several cells but rather to a real increase in the amplitude recorded from a particular neuron.

The porous nature of CNTs together with the fact that both the signal-to-noise ratio and the signal amplitudes are larger in CNT electrodes than in TiN electrodes led us to assume that the coupling between the tissue and the electrodes may improve with time on CNTs as the retina settles on the MEA and retinal cells become more intimately intermingled with the CNTs. This would be indicated by a gradual increase in spike amplitudes. To test this possibility, we have recorded signals at discrete intervals after tissue mounting on the electrodes, with the first sample always recorded within minutes after the beginning of the experiment, as soon as spontaneous activity becomes visible on the MEA. For both types of electrodes, the number of electrodes exhibiting neural activity generally increased with time. This is probably due both to tissue recovery from isolation from the eye and to improvement in coupling. However, signals recorded from TiN electrodes stabilized relatively quickly after tissue mounting whereas those recorded with CNT electrodes continued improving with time. Figure 4 illustrates the gradual increase in signal amplitude for two CNT electrodes (from the same MEA). The vertical axis represents spike amplitudes and the five panels respectively illustrate the recordings obtained from the electrodes at five consecutive time intervals. The strength of the bar shading on the graphs is proportional to the probability of recording and detecting spikes of given amplitude. In both cases there is a conspicuous increase in signal amplitude with time. The increase was already visible within $22 \mathrm{~min}$ from mounting for both electrodes. For electrode 75 (Figure 4A), there was a gradual increase in amplitudes for spikes below $100 \mu \mathrm{V}$ in subsequent samplings, as indicated by a downwards shift of high probabilities (dark shading). In addition to these changes, a new population of amplitudes ranging between 150 and $200 \mu \mathrm{V}$ emerged at 47 -min post mounting. The probability of occurrence of these larger spikes was low, as indicated by the light shading on the graph (marked by asterisks for easier detection). For electrode 83 (Figure 4B), the amplitude group around $100 \mu \mathrm{V}$ at the time of mounting clearly separated from the lower amplitudes in subsequent sampling periods, shifting to $150-200 \mu \mathrm{V}$ at 22-min 

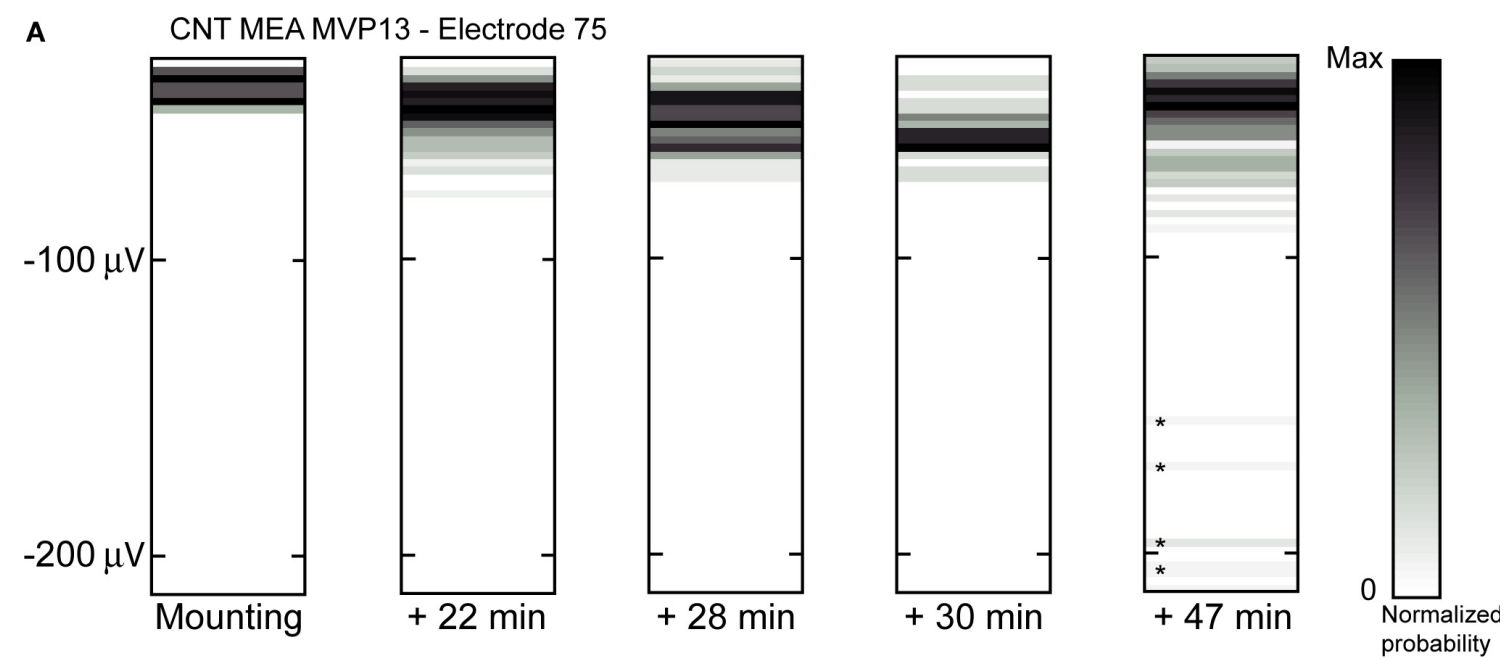

B $\quad$ CNT MEA MVP13 - Electrode 83
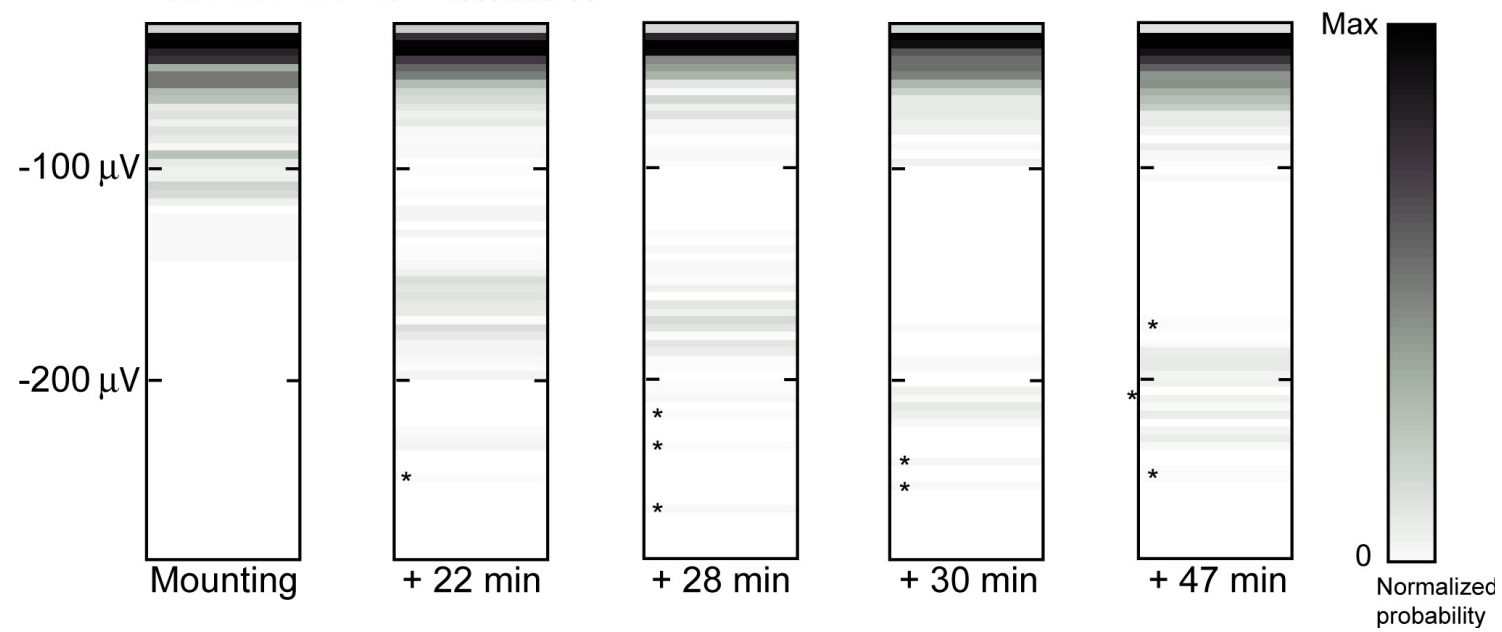

FIGURE 4 | Increase in spike amplitudes over time after retinal mounting on CNT electrodes. $(\mathbf{A}, \mathbf{B})$ Show two examples of amplitude changes recorded at increasing times after mounting (as indicated below the boxes) on two different electrodes from the same MEA. The vertical positions of the shaded bars in each box indicate the spike amplitudes whereas the shading itself indicates the probability of recording a spike at a given amplitude, with darker shading for higher probabilities. Asterisks indicate the positions of bars that are particularly difficult to detect because of the low probability of spike occurrence. post-mounting. In later sampling periods, this same group of amplitudes increased to values above $200 \mu \mathrm{V}$.

To systematically compare this effect for CNT and commercial MEAs, we have quantified the increase in signal amplitude for many electrodes in the following way. For each electrode on the MEA, the mean of the $25 \%$ highest spike amplitudes within the whole amplitude distribution was calculated for each one of the sampling periods. A linear approximation was then found between those means and the corresponding starting times of the sampling periods. The slope of this approximation was normalized to the mean of the first sampling period in order to obtain a growth rate parameter $(\% / \mathrm{min})$ to quantify the changes in amplitude with time.

We have calculated the spike amplitude growth rate for several MEAs, both with TiN and with CNT electrodes. Figure 5 illustrates the comparison in growth rate between a TiN MEA (Figure 5A) and a CNT MEA (Figure 5B). In nearly all cases, spikes recorded with CNT MEAs exhibited a clear positive amplitude growth rate over the sampling period. Spikes recorded with TiN MEAs, on the other hand, did not behave in a similar way. There was no conspicuous spike amplitude growth as a function of time with these electrodes. For the TiN MEA, values remain around 0, whereas for the CNT MEA, there is a clear growth in amplitudes, with growth rates of up to $2 \%$ per minute.

The superiority of CNT electrodes for stimulation applications have already demonstrated in an earlier study (Wang et al., 2006). We performed a preliminary investigation to validate the suitability of our CNT electrodes as an interface for retinal stimulation. Using an 8-channel STG stimulating system from MultiChannel Systems, we have stimulated individual electrodes $(80 \mu \mathrm{m}$ in diameter) on the MEA and recorded evoked spikes on neighboring electrodes (200 $\mu \mathrm{m}$ spacing). The stimulus applied were cathodic charge-balanced bi-phasic square current waveforms of various amplitudes and durations ranging from 50-700 $\mu \mathrm{A}$ and $60-100 \mu \mathrm{s}$ per phase respectively. At $480 \mu \mathrm{A}$, a $60-\mu$ s pulse (corresponding 


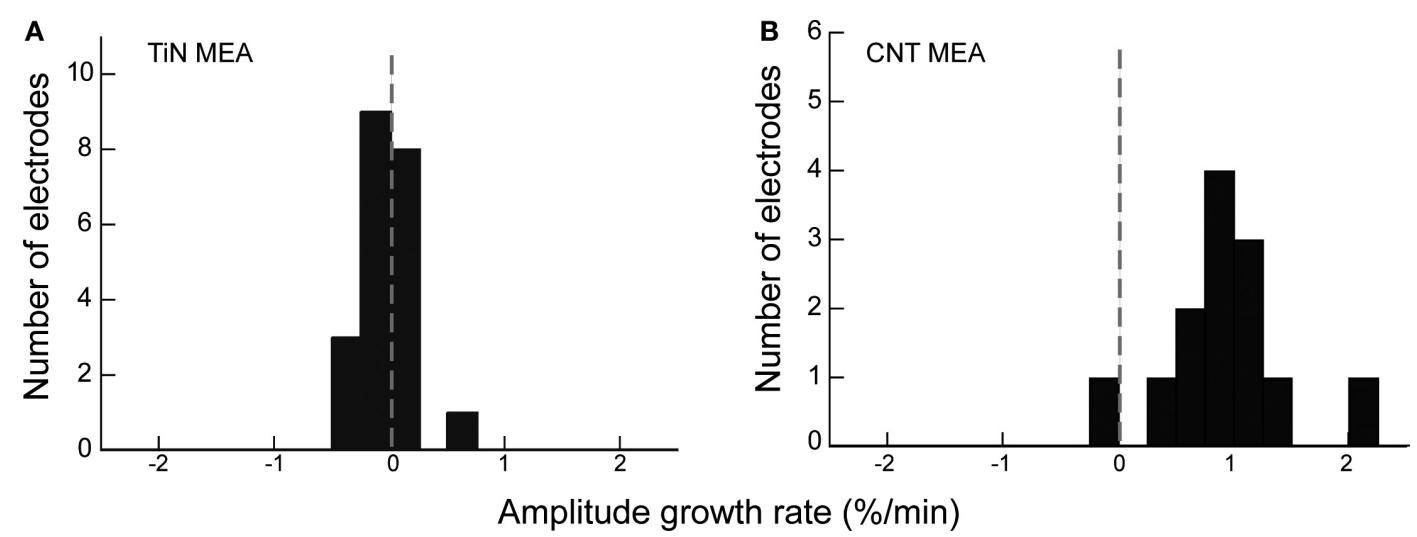

FIGURE 5 | Comparison of spike amplitude growth rate between TiN and CNT electrodes. (A) A histogram of amplitude growth rate for all electrodes on a TiN MEA. The dotted grey line indicates 0 growth rate. (B) A histogram of amplitude growth rate for all electrodes on a CNT MEA. There is a clear positive growth rate for the CNT electrodes, but not for the TiN electrodes.

to $28 \mathrm{nC}$ ) yielded responses in $16 \%$ of trials. Responses were generally observed on a single neighboring electrode. For $80 \mu \mathrm{s}$ pulses $(38 \mathrm{nC})$, responses were elicited in $75 \%$ of trials, and finally, responses were obtained in $100 \%$ trials for $100 \mu$ s pulses $(48 \mathrm{nC})$. As these tests were performed with recording electrodes located away from the stimulated electrodes, these results only provide a proof of concept to the ability to effectively use CNT electrodes for stimulations. The values reported here are comparable to previously reported data (Ahuja et al., 2008).

\section{DISCUSSION}

Here we have demonstrated the use of CNT micro electrodes as an improved scheme to record from the retina. Overall, our findings provide very compelling evidence that CNT-based electrodes are the way forward to improve the biocompatibility and durability of retinal prosthetic devices. Indeed, our observations demonstrate that CNT-based electrodes offer excellent electrochemical and adhesive properties, which could potentially make this material the ideal choice for long-term in vivo implantation.

The most conspicuous attribute of the CNT electrode compared with their more conventional counterparts is the improved signal to noise ratio achieved during recordings and an apparent increase in the signal amplitude over several hours after recording had begun. Since there is no change in waveform shape over time, we attribute the increase in recorded signal to improved coupling between the tissue and the electrode. It is conceivable to associate the increase in the recorded signal with tissue growth (Leng et al., 2004; Winter et al., 2008). Otherwise improvement in the mechanical coupling between the tissue and the electrodes through mere sinking of the tissue onto the CNTs may explain the improved interfacing. In the latter case the three-dimensional nature of the CNT matrix may contribute to better recorded signal due to a better (larger) contact between the cells and their processes and the electrodes. Penetration of CNTs into neurons may also give rise to improved signal to noise. However, it is unlikely that tubes have actually penetrated through the membrane as we rarely saw changes in signal shape. CNT penetration may result in intracellular-like action potentials, a phenomenon we have never observed.
Long-term studies and careful examination of the electrode-tissue interface will help to elucidate this issue.

The affinity of neuronal cells to adhere and entangle into the three-dimensional matrix of the CNTs has been studied by us extensively (Sorkin et al., 2009) with dissociated cells. This is a relatively rapid process in which dissociated cells can regenerate processes which readily intertwine with the CNTs. It remains to be investigated whether a related process takes place in the retina. We intend to study these effects with organotypic cultures of retinal explants. The three dimensional structure of the CNT electrodes and the manner by which neurites intertwine with the electrode surface is one of the most important features of these electrodes. Due to this intertwining, glial cells are less prone to block the interface between the electrode and the cells. We have directly demonstrated this effect with cultured cells (see Shein et al., 2009; Sorkin et al., 2009). As glia "scaring" is amongst the most challenging aspects in the design of high efficacy neural implant electrodes it is important to validate this effect with long-term, organotypic retinal cultures.

As CNT electrodes are gaining increased attention as novel neuronal interfaces, understanding the manner by which they interact or affect the activity of the overlying neurons is becoming important. One issue of increased attention is the nature of the electrical interface between the CNTs and the neurons. The data presented here is overall consistent with an improved capacitive coupling between the electrodes and the neurons. The vast majority of recorded signals have a similar shape, typically observed in extracellular recordings. On very rare occasions, atypical spikes were recorded (Fromherz et al., 1991). This effect will be further investigated and analyzed in the future.

An additional issue is whether CNTs affect the levels of neuronal firing. A recent investigation has demonstrated qualitative differences between the activity of neurons cultured on CNTs and on smooth surfaces (Lovat et al., 2005). Overall these conspicuous differences may be related to various parameters affecting cellular activity such as cell density, cell morphology, and mechanical tension to name just a few. Our data show no conspicuous increase in the firing rate of neurons on CNTs. As a matter of fact, in the examples shown in Figure 3, neural activity was stronger on the 
TiN electrodes. Overall, we did not observe significant differences in neural activity levels in TiN versus CNT MEAs. As our investigation involved only short term recordings (several hours) it is possible that additional processes take place at later stages. These effects will be explored using organotypic retinal cultures. It is important to note that culturing whole retinal explants will provide a reliable mean to study the effect of CNTs on neurons within their natural microenvironment (rather than in dissociated cultures). Indeed, the retina is a highly organized system, with a well defined cellular arrangement in discrete layers, and therefore it will provide a powerful mean to explore brain-CNT interactions for future long-term neural implant applications.

Finally, as we have discussed in the introduction, the use of optimized MEAs for retinal stimulation is of great importance. An earlier

\section{REFERENCES}

Ahuja, A. K., Behrend, M. R. Kuroda, M., Humayun, M. S., and Weiland, J. D. (2008). An in vitro model of a retinal prosthesis. IEEE Trans. Biomed. Eng. $55,1744-1753$.

den Hollander, A., Roepman, R., Koenekoop, R.K., and Cremers, F. P. M. (2008). Leber congenital amaurosis: genes, proteins and disease mechanisms. Prog. Retin. Eye Res. 27, 391-419.

Dowling, J. (2008). Current and future prospects for optoelectronic retinal prostheses. Eye (Cambridge Ophthalmological Symposium) 22, 1-7.

Ehrlich, R., Harris, A., Kheradiya, N. S., Winston, D. M., Ciulla, T. A., and Wirostko, B. (2008). Age-related macular degeneration and the aging eye. Clin. Interv. Aging 3, 473-482.

Fromherz, P., Offenhausser, A., Vetter, T., and Weis, J. (1991). A neuron-silicon junction: a Retzius cell of the leech on an insulated-gate field-effect transistor. Science 252, 1290-1293.

Gabay, T., Ben-David, M., Kalifa, I., Sorkin, R., Abrams, Z. R., BenJacob, E., and Hanein, Y. (2007). Electro-chemical and biological properties of carbon nanotube based multielectrode arrays. Nanotechnology 5, 035201-035206.

Gunning, D., Adams, C., Cunningham, W., Mathieson, K.,O'Shea, V.,Smith, K. M., Chichilnisky, E. J., Litke, A. M., and Rahman, M. (2005). $30 \mu \mathrm{m}$ Spacing 519-electrode arrays for in vitro retinal studies. Nucl. Instrum. Methods Phys. Res. A 546, 158-153.

Hennig, M. H., Adams, C., Willshaw, D., and Sernagor, E. (2009). Early-stage waves in the retinal network emerge close to a critical state transition between local and global functional connectivity. J. Neurosci. 29, 1077-1086.

Hu,H.,Ni,Y.C., Mandal,S.K.,Montana, V., Zhao, N., Haddon, R. C. et al. (2005). Polyethyleneimine functionalized single-walled carbon nanotubes as a substrate for neuronal growth. J. Phys. Chem. B 109, 4285-4289.

Hu, H., Ni, Y. C., Montana, V., Haddon, R. C., and Parpura, V. (2004). Chemically functionalized carbon nanotubes as substrates for neuronal growth. Nano Lett. 4, 507-511.

Hulata, E., Segev, R., and Ben-Jacob, E. detection based on wavelet packets and Shannon's mutual information. J. Neurosci. Methods. 117, 1-12.

Janders, M., Egert, U., Stelzle, M., and Nisch, W. (1996). Novel Thin Film Titanium Nitride Micro-Electrodes with Excellent Charge Transfer Capability for Cell Stimulation and Sensing Applications, Engineering in Medicine and Biology Society, 1996 Bridging Disciplines for Biomedicine. Proceedings of the Annual International Conference of the IEEE 1, 245-247.

Leng, T., Wu, P., Mehenti, N.Z., Bent, S. F., Marmor, M. F., Blumenkranz, M. S., and Fishman, H. A. (2004). Directed retinal nerve cell growth for use in a retinal prosthesis interface. Invest. Ophthalmol. Vis. Sci. 45, 4132-4137.

Lovat, V., Pantarotto, D., Lagostena, L., Cacciari, B., Grandolfo, M., Righi, M., Spalluto, G., Prato, M., and Ballerini, L. (2005). Carbon nanotube substrates boost neuronal electrical signaling. Nano Lett. 5-6, 1107-1110.

Mahadevappa, M., Weiland, J. D., Yanai, D., Fine, I., Greenberg, R. J., and Humayun, M. S. (2005). Perceptual thresholds and electrode impedance (2002). A method for spike sorting and

study by Wang et al. (2006) has already validated the effectiveness of CNT electrodes for stimulation applications. The advantages offered by CNT electrodes are stemming from their improved electrochemical capacitance, and accordingly, their improved charge injection limit. We plan to further validate this issue using organotypic retinal cultures, towards the formation of a superior MEA-retina interface.

\section{ACKNOWLEDGEMENTS}

The TAU CNT-MEA project was partially supported by an ISF grant and a grant from the TAU Adams Super Center for Brain Studies. The NCL retinal project is funded by the Centre of Excellence for Life Sciences the EPSRC, BBSRC and Newcastle University Hospitals Special Trustees.

in three retinal prosthesis subjects. IEEE Trans. Neural Syst. Rehabil. Eng. 13, 201-206.

Malarkey, E. B., and Parpura, V. (2007). Applications of carbon nanotubes in neurobiology. Neurodegener. Dis. 4 292-299.

Mattson, M. P., Haddon, R. C., and Rao, A. M. (2000). Molecular functionalization of carbon nanotubes and use as substrates for neuronal growth J. Mol. Neurosci. 14, 175-182.

Rizzo, J. F., Snebold, L., and Kenney, M. (2007). Development of a visual prosthesis. In Visual Prosthesis and Ophthalmic Devices: New Hope in Sight, J. Tombran-Tink, C. Barnstable, and J. F. Rizzo, eds (Totawa, NJ Humana Press), pp 71-93.

Rizzo, J. F., Wyatt, J., Loewenstein, J., Kelly, S., and Shire, D. (2003). Methods and perceptual thresholds for short-term electrical stimulation of human retina with microelectrode arrays. Invest. Ophthalmol. Vis. Sci. 44 5355-5361.

Sekirnjak, C., Hottowy, P., Sher, A., Dabrowski, W., Litke, A. M., and Chichilnisky, E. J. (2006). Electrical stimulation of mammalian retinal ganglion cells with multielectrode arrays. J. Neurophysiol. 95, 3311-3327.

Sernagor, E., Eglen, S. J., and Wong, R. O. L. (2001). Development of retinal ganglion cell structure and function. Prog. Retin. Eye Res. 20, 139-174.

Shein, M., Greenbaum, A., Gabay, T. Sorkin, R., David-Pur, M., BenJacob, E., and Hanein, Y. (2009). Engineered neuronal circuits shaped and interfaced with carbon nanotube microelectrode arrays. Biomed. Microdevices. 11, 495-501.

Sorkin, R., Gabay, T., Blinder, P., Baranes, D., Ben-Jacob, E., and Hanein,
Y. (2006). Compact self-wiring in cultured neural networks. J. Neural Eng. 3, 95-101.

Sorkin, R., Greenbaum, A., David-Pur, M., Anava, S., Ayali, A., Ben-Jacob, E., and Hanein, Y. (2009). Process entanglement as a neuronal anchorage mechanism to rough surfaces. Nanotechnology 20, 015101-015108.

Wang, K., Fishman, H. A., Dai, H., and Harris, J.S. (2006). Neural stimulation with carbon nanotube microelectrode array. Nano Lett. 6, 2043-2048.

Winter, J. O., Gokhaleb, M., Jensena, R. J., Cogand, S. F., and Rizzo, J. F. (2008). Tissue engineering applied to the retinal prosthesis: neurotrophin-eluting polymeric hydrogel coatings. Mater. Sci. Eng. C 28, 448-453.

Conflict of Interest Statement: The authors declare that the research was conducted in the absence of any commercial or financial relationships that could be construed as a potential conflict of interest.

Received: 11 February 2009; paper pending published: 02 March 2009; accepted: 03 April 2009; published: 20 April 2009 Citation: Shoval A, Adams C, David-Pur M, Shein M, Hanein, Y. and Sernagor, E. (2009) Carbon nanotube electrodes for effective interfacing with retinal tissue. Front. Neuroeng. (2009) 2:4. doi:10.3389/neuro.16.004.2009

Copyright (c) 2009 Shoval, Adams, David-Pur, Shein, Hanein and Sernagor. This is an open-access article subject to an exclusive license agreement between the authors and the Frontiers Research Foundation, which permits unrestricted use, distribution, and reproduction in any medium, provided the original authors and source are credited. 